\title{
Optical Response of fci-ZnMgHo Quasicrystal
}

\author{
V. Karpus ${ }^{a, *}$, G.-J. Babonas ${ }^{a}$, A. Réza ${ }^{a}$, A. Suchodolskis $^{a}$,

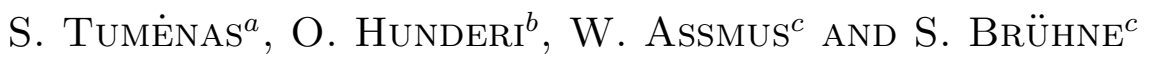 \\ ${ }^{a}$ Semiconductor Physics Institute \\ A. Goštauto 11, 01108 Vilnius, Lithuania \\ ${ }^{b}$ NTNU, Høgskoleringen 5, 7491 Trondheim, Norway \\ ${ }^{c}$ J.W. Goethe-Universität \\ Max-von-Laue-Str. 1, 60438 Frankfurt am Main, Germany \\ The results of an optical spectroscopy study of the icosahedral \\ fci-ZnMgHo quasicrystal are presented. The dielectric function of the qua- \\ sicrystal was measured by the spectroscopic ellipsometry technique in the \\ spectral range of $0.1-5.0 \mathrm{eV}$. The analysis of the dielectric function shows \\ that an optical response of fci- $\mathrm{ZnMgHo}$ is a superposition of the free-electron \\ Drude-type contribution and that of the interband transitions across the fci- \\ $\mathrm{ZnMgHo}$ pseudogap.
}

PACS numbers: $71.23 . \mathrm{Ft}$

\section{Introduction}

The electronic structure of face-centred icosahedral (fci) ZnMgRE (RE = $\mathrm{Y}, \mathrm{Ho}, \mathrm{Er})$ quasicrystals was recently examined by the photoemission (PE) spectroscopy technique $[1,2]$. Analysis of the PE response reveals that the electron energy spectrum of the quasicrystals near the Fermi level is determined by intersections of the isoenergetic surface with the 222100 and 311111 Bragg planes, which make up the effective Brillouin zone.

In the present paper we report on the fci-ZnMgHo optical study, carried out by the spectroscopic ellipsometry technique. The analysis of optical spectra shows that the fci-ZnMgHo optical response in general trends can be synthesised by the same model of the electronic structure, which was derived in analysis of PE response of the quasicrystal.

\section{Experimental}

The fci- $\mathrm{Zn}_{65} \mathrm{Mg}_{25} \mathrm{Ho}_{10}$ single-grain quasicrystals were grown by the liquidencapsulated top-seeded solution-growth method [3]. The optical surface of sam-

*corresponding author; e-mail: karpus@pfi.lt 
ples was prepared by mechanical polishing with finely grained, $1 \mu \mathrm{m}$ and $0.25 \mu \mathrm{m}$, diamond paste. Optical measurements of the fci- $\mathrm{ZnMgHo}$ dielectric function were carried out by the spectroscopic ellipsometry technique. The ellipsometric measurements in the spectral ranges of $0.5-5 \mathrm{eV}$ and $0.1-0.5 \mathrm{eV}$ were carried out, correspondingly, by the photometric ellipsometer with rotating analyser and by IR ellipsometer equipped with the Fourier transform spectrometer.

\section{Results and discussion}

The experimental spectra of the fci-ZnMgHo dielectric function $\varepsilon(\omega)$ are presented in Fig. 1. The middle-IR spectral features at 0.18 and $0.29 \mathrm{eV}$ are most probably artefacts due to residual gasses in ellipsometer chamber. The $\varepsilon(\omega)$ dispersion is close to that measured previously for fci-ZnMgY and fci-ZnMgEr quasicrystals $[4,5]$. The dielectric function presents a superposition of the free-electron Drude-type optical response and a contribution of the interband transitions. The latter obviously manifests itself in the real part of $\varepsilon(\omega)$, which has a spectral feature at about $1 \mathrm{eV}$.

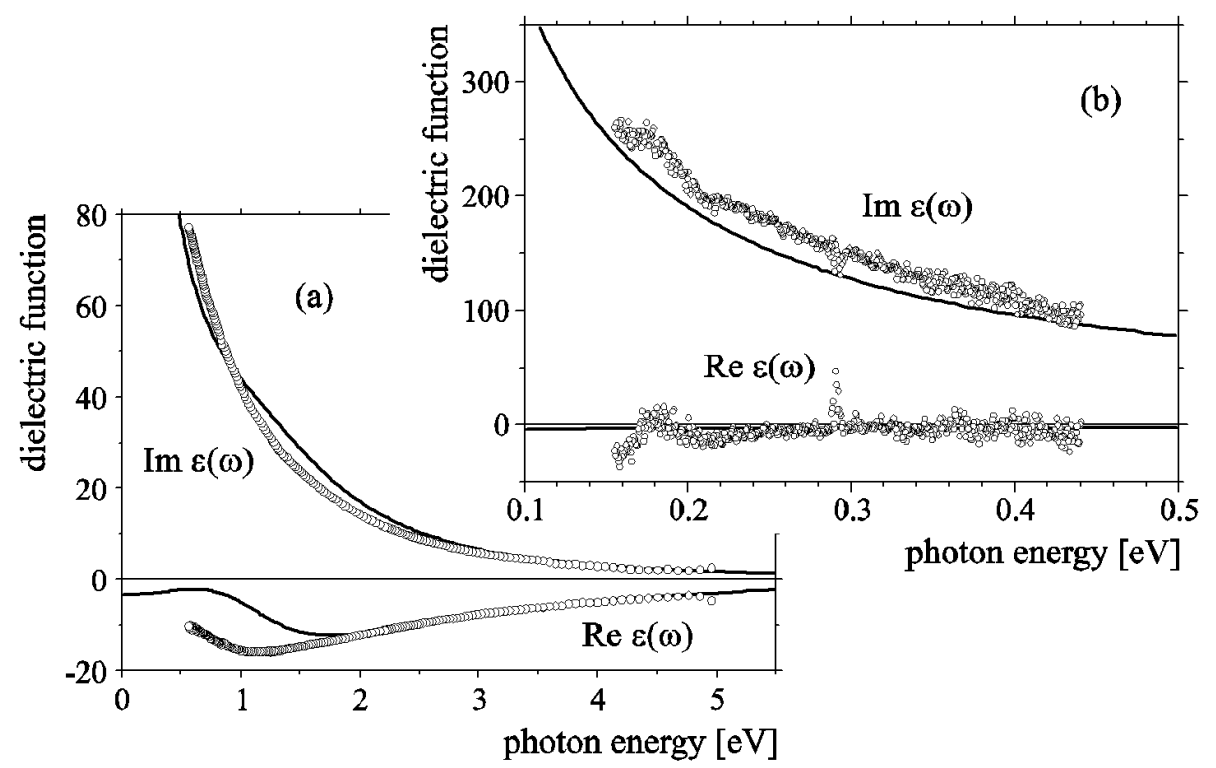

Fig. 1. Dispersion of the dielectric function of fci-ZnMgHo quasicrystal in the near-IRUV (a) and middle-IR regions (b). Dots and curves present experimental and theoretical data, respectively.

To analyse an optical response of metallic solids, it is convenient to express the dielectric function $\varepsilon(\omega)$ via the optical conductivity $\sigma(\omega)$,

$$
\varepsilon(\omega)=1+\mathrm{i} \frac{4 \pi}{\omega} \sigma(\omega), \quad \sigma(\omega)=\sigma_{\text {Drude }}(\omega)+\sigma_{\mathrm{ib}}(\omega) .
$$

Here $\sigma_{\text {Drude }}(\omega)$ is the free-electron optical conductivity and $\sigma_{\mathrm{ib}}(\omega)$ is the optical 
conductivity due to the interband transitions. The $\sigma_{\text {Drude }}(\omega)$ term, according to the Drude formula $\sigma_{\text {Drude }}(\omega)=\sigma_{\mathrm{DC}} /(1-\mathrm{i} \omega \tau)$, is defined by the static electric conductivity $\sigma_{\mathrm{DC}}$ and the relaxation time $\tau$. The $\sigma_{\mathrm{ib}}(\omega)$ term due to the interband transitions across a pseudogap can be calculated within the framework of nearlyfree electronic model following Ashcroft and Sturm algorithm [6]. The calculations require for a given set of $g, \Delta, \varepsilon_{\mathrm{F}}$, and $\Gamma$ parameters, where $g$ is the reciprocal lattice vector of the Bragg plane, which intersects an isoenergetic surface, $\Delta$ is the corresponding pseudogap, $\varepsilon_{\mathrm{F}}$ is the Fermi energy, and $\Gamma$ is the broadening parameter. According to the fci- $\mathrm{ZnMgHo}$ electronic structure model, deduced in analysis of photoemission response [1], the Fermi level is located above the $\Delta_{211000}$ pseudogap and lies within the $\Delta_{311111}$ pseudogap. Therefore, one can expect that the interband optical transitions first of all are due to $\Delta_{311111}$ pseudogap, the corresponding $g$-vector of which is of $3.18 \times 10^{8} \mathrm{~cm}^{-1}$.

Results of the optical response (1) simulations, under the assumption of the interband transitions across the $\Delta_{311111}$ pseudogap, are presented by curves in Fig. 1 and Fig. 2. As seen, they qualitatively reproduce the experimental $\varepsilon(\omega)$ and $\sigma(\omega)$ dispersions. It is important that the parameters of electron energy

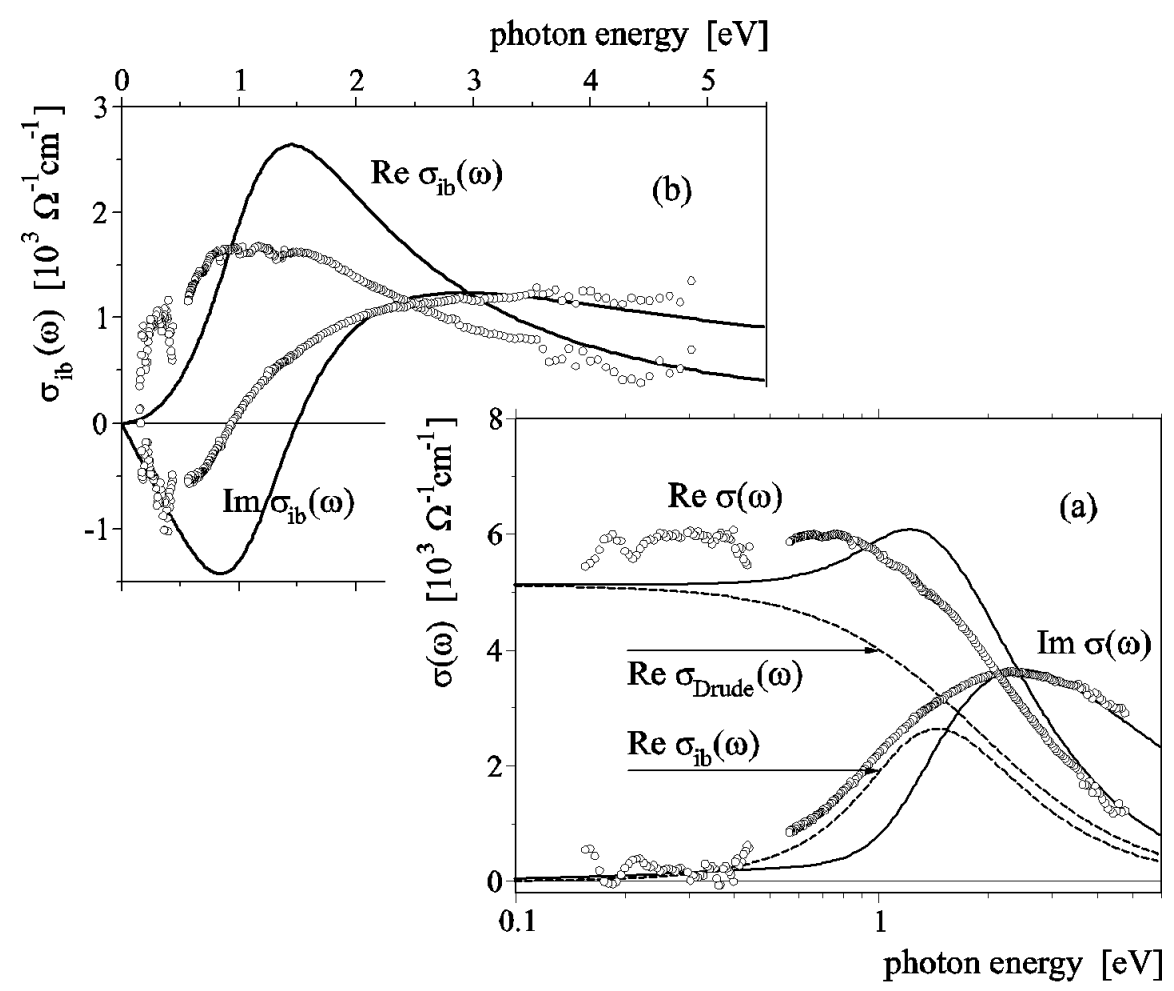

Fig. 2. Optical conductivity of fci-ZnMgHo quasicrystal. Dots and curves present experimental and theoretical data, respectively (dots in (b) were obtained by subtraction of determined Drude contribution from experimental $\sigma(\omega)$ data). 
spectrum, $\Delta_{311111}=1 \mathrm{eV}, \varepsilon_{\mathrm{F}}=9.55 \mathrm{eV}, \Gamma=0.64 \mathrm{eV}$, deduced by the leastsquares fit of optical data, are close to those, determined in PE study [1]: $\Delta_{311111}=$ $0.98 \mathrm{eV}, \varepsilon_{\mathrm{F}}=9.32 \mathrm{eV}, \Gamma=0.22 \mathrm{eV}$. This is a sound support of the suggested model of the fci-ZnMgHo electronic structure. The determined parameters of the free-electron optical response, $\sigma_{\mathrm{DC}}=5130 \Omega^{-1} \mathrm{~cm}^{-1}$ and $\hbar / \tau=1.9 \mathrm{eV}$, are in agreement with the static conductivity value of $5410 \Omega^{-1} \mathrm{~cm}^{-1}$, recorded by electrical measurements [7], and with the large scattering parameter value of $\hbar / \tau \approx 1 \mathrm{eV}$, which is a generic characteristic of all quasicrystals. The estimated value of the fci-ZnMgHo plasma frequency $\hbar \omega_{\mathrm{p}} \approx 11 \mathrm{eV}$ is comparable with the $\hbar \omega_{\mathrm{p}} \approx 8.3 \mathrm{eV}$ value of fci-ZnMgY and fci-ZnMgTb, determined by Chernikov et al. [8] from an analysis of reflectivity spectra of the quasicrystals.

Summarizing, we conclude that the fci- $\mathrm{ZnMgHo}$ optical response presents a superposition of the free-electron and interband contributions. The optical data can be interpreted by the electron energy structure model, according to which the electron energy spectrum in a vicinity of the Fermi level is determined by intersections of the isoenergetic surface with an effective Brillouin zone.

\section{Acknowledgments}

Support by the Research Council of Norway is gratefully acknowledged.

\section{References}

[1] A. Suchodolskis, W. Assmus, L. Giovanelli, U.O. Karlsson, V. Karpus, G. Le Lay, R. Sterzel, E. Uhrig, Phys. Rev. B 68, 054207 (2003).

[2] A. Suchodolskis, W. Assmus, L. Giovanelli, U.O. Karlsson, V. Karpus, G. Le Lay, E. Uhrig, J. Phys., Condens. Matter 16, 9137 (2004).

[3] A. Langsdorf, W. Assmus, J. Cryst. Growth 192, 152 (1998).

[4] V. Karpus, G.-J. Babonas, A. Rèza, W. Assmus, R. Sterzel, Lith. J. Phys. 40, 118 (2000).

[5] A. Suchodolskis, W. Assmus, G.-J. Babonas, L. Giovanelli, U.O. Karlsson, V. Karpus, G. Le Lay, A. Rèza, E. Uhrig, Acta Phys. Pol. A 107, 412 (2005).

[6] N.W. Ashcroft, K. Sturm, Phys. Rev. B 3, 1898 (1971).

[7] I.R. Fisher, Z. Islam, A.F. Panchula, K.O. Cheon, M.J. Kramer, P.C. Canfield, A.I. Goldman, Philos. Mag. B 77, 1601 (1998).

[8] M.A. Chernikov, S. Paschen, E. Felder, P. Vorburger, B. Ruzicka, L. Degiorgi, H.R. Ott, I.R. Fisher, P.C. Canfield, Phys. Rev. B 62, 262 (2000). 